\title{
Vibration Characteristics of Grouped Steam Turbine Blades*
}

\begin{abstract}
Motohiro SHIGA**
The resonant amplitudes of grouped turbine blades were experimentally measured and theoretically calculated to determine the difference between calculated and experimental values. The blades were excited by an electromagnet, and the calculated values of the resonant amplitude distribution at the natural frequency were found to agree with the measured values. At some measuring points, a difference occurred between the calculated values and the measured ones, and in the worst case, the measured values became about twice the calculated ones. The calculated values also agreed well with the measured ones with regard to the direction of vibration and resonant stress. Therefore, if the operational exciting force can be estimated when designing the blades, the vibration amplitudes and stresses of the grouped blades can be calculated with some certainty. This is very useful for producing well-designed grouped blades.
\end{abstract}

Key Words: Vibration, Steam Turbine Blade, Vibration Characteristics, Binded Blades

\section{Introduction}

Blades of a steam turbine are subjected to periodic forces with frequencies equal to the rotational speed or its harmonics due to an imbalance in steam flow. If the exciting frequency coincides with one of the natural frequencies of the blades, a resonant stress will occur in the blade, possibly causing damage to the blade. Many calculation methods of blade natural frequencies have been developed; one of these is able to predict the natural frequencies when designing a steam turbine (1)(2) $^{2}$

A steam turbine used in a power plant is operated at a constant rotational speed. Therefore, if resonance does not occur at the rated speed, vibration is unlikely to cause damage to the blades. However, because the steam turbine may pass through a resonance speed during starting or stopping, it is necessary to calculate vibration amplitudes and stresses on the blades during resonance.

* Received 12th May, 1989. Paper No. 88-0533B

* Mechanical Engineering Research Laboratory, Hitachi Ltd., Kandatsu, Tsuchiura, Ibaraki, 300, Japan
If the vibration force acts on the grouped blades, the resonant amplitude and stress can be calculated. So far, results of the comparisons between calculated and measured values have not been well reported. However, it is important for the designer of steam turbine blades to investigate how far the calculated values deviate from the measured results.

When a steam turbine rotates, a vibrational force acts on all grouped blades. If the calculated vibration response characteristics agree with the measured values in the stationary state, the vibration response in the rotating state can be predicted by use of vibration analysis ${ }^{(1)}$. In this method the blades are divided and the relationships between the displacement, the slope, the moment and the shearing force with regard to the each element are determined. From those relationships the frequency equation is determined. The effect of centrifugal force due to rotation, is considered in calculation by a force acting in the longitudi. nal direction of the blade. If the vibration amplitude is determined when the exciting force acts at a point, the vibration amplitude when the exciting force acts on all grouped blades can be obtained by summing the vibration amplitudes resulting from many exciting 
forces. This is the basis for predicting the vibration characteristics of grouped blades to obtain the calculated and measured vibration amplitudes of grouped blades. The calculated amplitude values were compared to the measured ones by exciting a point on the grouped blades using an electromagnet.

\section{Calculation Method}

\section{1 Resonant amplitude of the grouped blades}

The excited position and vibration-measuring position are shown in Fig. 1. The excited position is A, and the measuring position is $B$. The exciting force at position $\mathrm{A}$ is $F_{a} \cos \omega t$, the displacement of that position is $V_{A} \sin \omega t$, and the displacement of the measured position $B$ is $V_{B} \sin \omega t$.

The supplied energy ${ }^{(3)}, E_{s}$, at A during one cycle of vibration is:

$$
E_{s}=\pi F_{a} V_{A} \text {. }
$$

The energy, $E_{d}$, which is consumed by damping, is twice the product of all vibration energies of the grouped blades and logarithmic decrement ${ }^{(3)}$. The result is as follows:

$$
E_{d}=2 \delta\left[\Sigma \frac{1}{2} m \omega^{2} Y^{2}+\Sigma \frac{1}{2} J \omega^{2} \Phi^{2}\right],
$$

where

$\delta:$ logarithmic decrement

$Y$ : displacement amplitude

$m$ : mass

$J$ : moment of inertia

$\omega:$ circular frequency

$\Phi$ : amplitude of torsional vibration.

In calculating the vibration of the grouped blades, the tangential component, $Y_{t}$, of the amplitude and the axial component, $Y_{a}$, are calculated separately. In such a case, $Y_{t}^{2}+Y_{a}^{2}$ is used instead of $Y^{2}$ in Eq. (2).

As energy, $E_{s}$, which is supplied by the exciting force in the resonant condition, is equal to the energy, $E_{d}$, consumed by damping, we obtain the following equations :

$$
\begin{aligned}
& \pi F_{a} V_{A}=\delta \omega^{2}\left[\sum m Y^{2}+\sum J \Phi^{2}\right], \\
& \pi F_{a} V_{A}=\delta \omega^{2} V_{A}^{2}\left(\frac{Y_{m}}{V_{A}}\right)^{2}\left[\sum m\left(\frac{Y}{Y_{m}}\right)^{2}+\sum J\left(\frac{\Phi}{Y_{m}}\right)^{2}\right],
\end{aligned}
$$

where

$Y_{m}$ : maximum value of displacement amplitude.

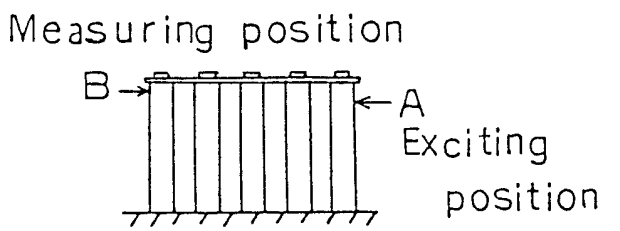

Fig. 1 Exciting position and measuring position
The values of $Y_{m} / V_{A}, \Sigma m\left(Y / Y_{m}\right)^{2}+\Sigma J\left(\Phi / Y_{m}\right)^{2}$ in Eq. (3) are determined by the calculation of the natural frequency and vibration mode of the grouped blades. $V_{A}$ is obtained from these values, and the result is as follows:

$$
\left.\begin{array}{l}
V_{A}=\frac{\pi F_{a}}{\delta \omega^{2}}\left(\frac{V_{A}}{Y_{m}}\right)^{2} \frac{1}{G}, \\
G=\sum m\left(\frac{Y}{Y_{m}}\right)^{2}+\Sigma J\left(\frac{\Phi}{Y_{m}}\right)^{2} .
\end{array}\right\}
$$

The amplitude, $V_{B}$, at measuring position $\mathrm{B}$ becomes :

$$
V_{B}=\left(V_{B}^{\prime} / V_{A}^{\prime}\right) V_{A},
$$

where $V_{B}^{\prime} / V_{A}^{\prime}$ is the amplitude ratio at $\mathrm{A}$ and $\mathrm{B}$, which is determined by the calculation of the vibration mode.

$V_{A} / Y_{m}, \sum m\left(Y / Y_{m}\right)^{2}+\sum J\left(\Phi / Y_{m}\right)^{2}$ in Eq. (4) and $V_{B}{ }^{\prime} / V_{A}{ }^{\prime}$ in Eq. (5) are determined by the calculation of the natural frequency and vibration mode. These are also calculated by the vibration analysis method used for grouped blades ${ }^{(1)}$.

As the measurement of this experiment is done in the stationary state, the amplitude is calculated in the condition that the rotating speed is zero. The amplitude in the rotating state can be obtained by the use of values of $V_{A} / Y_{m}, \sum m\left(Y / Y_{m}\right)^{2}+\sum J\left(\Phi / Y_{m}\right)^{2}$ and $V_{B}{ }^{\prime} / V_{A}{ }^{\prime}$ corresponding to rotating condition.

\section{2 Exciting force}

The exciting force acting on the grouped blades as a result of the magnet was measured by setting a search coil on the electromagnet and measuring the voltage induced in the search coil.

Magnetic flux density of the magnet is $B(T)$, and the cross-sectional area of the magnetic path is $S_{m}$ $\left(\mathrm{m}^{2}\right)$. Therefore, the induced force, $F(\mathrm{~N})$, from the magnet becomes :

$$
F=\frac{10^{7}}{8 \pi} B^{2} S_{m}
$$

When a sine-wave electric current flows in the magnet, the magnetic flux density becomes $B=B_{a}$ sin $\omega t$. The induced force becomes:

$$
\begin{aligned}
F & =\frac{10^{7}}{8 \pi} S_{m} B_{a}^{2} \sin ^{2} \omega t=\frac{10^{7}}{8 \pi} S_{m} B_{a}{ }^{2}\left(\frac{1}{2}-\frac{1}{2} \cos 2 \omega t\right) \\
& =F_{0}-F_{1} \cos 2 \omega t .
\end{aligned}
$$

As the fluctuating component, $F_{1} \cos 2 \omega t$, of the force, $F$, is the exciting force, the magnitude, $F_{1}$, of the exciting force is :

$$
F_{1}=\frac{10^{7}}{16 \pi} S_{m} B_{a}{ }^{2}
$$

From the amplitude, $B_{a}$, of the fluctuating mag. netic flux density, the magnitude, $F_{1}$, of the exciting force can be calculated by Eq. ( 8 ).

The exciting force is represented by $F_{1} \cos 2 \omega t$. From this result, the exciting frequency becomes twice the frequency of the alternating current that flows in the magnet. 
The voltage, $e(\mathrm{~V})$, induced by the fluctuation of the magnetic flux, $\phi(\mathrm{Wb})$, becomes

$$
e=-d \phi / d t \text {. }
$$

The cross-sectional area of the search coil is $S\left(\mathrm{~m}^{2}\right)$, the number of turns of the search coil is $n$, and the magnetic flux density is $B_{a} \sin \omega t$. Then, the induced voltage, $e$, of the search coil is

$$
e=-\frac{d \phi}{d t}=-\frac{d}{d t}\left(\operatorname{Sn} B_{a} \sin \omega t\right)=-\operatorname{Sn} B_{a} \omega \cos \omega t .
$$

As the induced voltage of the search coil is represented by $e=-e_{m} \cos \omega t$, the amplitude, $B_{a}$, of the magnetic flux density becomes

$$
B_{a}=\frac{e_{m}}{S n \omega}=\frac{e_{m}}{2 \pi f_{1} S n},
$$

where $f_{1}$ is the frequency $(\mathrm{Hz})$ of the alternating current supplied in the magnet.

The amplitude, $B_{a}$, of the magnetic flux density can be determined by measuring the amplitude, $e_{m}$, of the induced voltage in the search coil.

\subsection{Logarithmic decrement}

By analyzing the relationship between the frequency and the amplitude, the logarithmic decrement, $\delta$, can be obtained by the following equation ${ }^{(4)}$ :

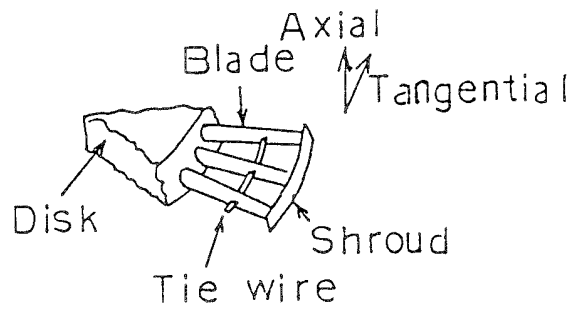

Fig. 2 Grouped turbine blade

$$
\hat{\delta}=\frac{\pi \Delta f}{f_{0}},
$$

where

$f_{0}$ : natural frequency $\mathrm{Hz}$

$\Delta f:$ the frequencies at which the amplitude becomes $1 / \sqrt{2}$ times the resonant amplitude exist before and after the natural frequency. If they are represented by $f_{a}$ and $f_{b}\left(f_{b}>f_{a}\right)$, then $\Delta f=f_{b}$ $-f_{a}$

\section{Test Results}

\section{1 Measured model}

The grouped turbine blades are shown in Fig. 2. The turbine blades are bound at the tips of several blades by shrouds and at the middle of the blades by tie wires. Such blades are called grouped blades.

The blade unit used in the test model has the same structure as a blade in an actual machine. That is, five actual blades are set on a model disk and fixed to the base by bolts. The five blades are bound by tie wires and shrouds.

In the vibration mode, the mode found at the lowest natural frequency of the grouped blades, the blade tips vibrate mainly in the tangential direction, that is, the rotating direction. Therefore, this natural frequency is called the tangential natural frequency.

At the next natural frequency, the blade tips vibrate mainly in the axial direction, and the five blades vibrate in phase. This natural frequency is called the axial natural frequency.

The resonant amplitude was calculated and mea-

\begin{tabular}{|c|c|c|c|c|c|}
\hline $\begin{array}{l}\text { Distance from } \\
\text { blade root } \\
\mathrm{cm}\end{array}$ & $\begin{array}{l}\text { Area of cross } \\
\text { section } \\
\mathrm{cm}^{2}\end{array}$ & $\begin{array}{l}\text { Maximum } \\
\text { moment of } \\
\text { inertia of } \\
\text { cross section } \\
\qquad \mathrm{cm}^{4}\end{array}$ & $\begin{array}{l}\text { Minimum } \\
\text { moment of } \\
\text { inertia of } \\
\text { cross section } \\
\mathrm{cm}^{4}\end{array}$ & $\begin{array}{l}\text { Direction of } \\
\text { principal } \\
\text { axis } \\
\text { degree }\end{array}$ & $\begin{array}{l}\text { Torsional } \\
\text { rigidity } \\
\text { factor } \\
\mathrm{cm}^{4}\end{array}$ \\
\hline $\begin{array}{l}0.0 \\
2.46 \\
4.94 \\
7.42 \\
10.06 \\
12.70 \\
15.88 \\
19.05 \\
22.2 \\
25.4 \\
29.2 \\
30.5 \\
31.8 \\
34.9 \\
38.1 \\
41.2 \\
44.2 \\
47.3 \\
50.8\end{array}$ & $\begin{array}{r}26.1 \\
9.63 \\
7.40 \\
5.88 \\
5.42 \\
4.99 \\
4.57 \\
4.15 \\
3.74 \\
3.33 \\
2.84 \\
3.84 \\
2.84 \\
2.57 \\
2.36 \\
2.24 \\
2.14 \\
2.08 \\
3.48\end{array}$ & $\begin{array}{r}239 \\
30.0 \\
21.7 \\
16.01 \\
14.19 \\
12.55 \\
11.11 \\
9.75 \\
8.48 \\
7.29 \\
5.98 \\
8.27 \\
5.98 \\
5.36 \\
4.84 \\
4.44 \\
4.15 \\
3.94 \\
6.87\end{array}$ & $\begin{array}{c}13.54 \\
4.35 \\
2.83 \\
1.795 \\
1.482 \\
1.211 \\
0.999 \\
0.806 \\
0.634 \\
0.482 \\
0.326 \\
0.468 \\
0.326 \\
0.249 \\
0.187 \\
0.148 \\
0.117 \\
0.093 \\
0.213\end{array}$ & $\begin{array}{r}0.0 \\
5.2 \\
6.6 \\
8.7 \\
11.8 \\
15.0 \\
18.2 \\
21.9 \\
26.1 \\
30.7 \\
37.1 \\
37.8 \\
37.1 \\
41.5 \\
45.6 \\
48.5 \\
51.8 \\
55.6 \\
60.5\end{array}$ & $\begin{array}{l}46.0 \\
6.18 \\
3.43 \\
1.660 \\
1.383 \\
1.140 \\
0.931 \\
0.744 \\
0.580 \\
0.437 \\
0.296 \\
0.686 \\
0.296 \\
0.233 \\
0.185 \\
0.159 \\
0.143 \\
0.135 \\
0.617\end{array}$ \\
\hline
\end{tabular}
sured with respect to the tangential and axial natural frequencies about a blade $50.8 \mathrm{~cm}$ long. The blade's

Table 1 Blade cross section data 
cross-sectional data are shown in Table 1 . The radius of the blade root is $50.8 \mathrm{~cm}$; the modulus of the longitudinal elasticity is $205.8 \mathrm{GPa}$; the modulus of the transverse elasticity is $82.32 \mathrm{GPa}$, and the density is $7850 \mathrm{~kg} / \mathrm{m}^{3}$. The tie wire is positioned $30.5 \mathrm{~cm}$ from the blade root; the cross-sectional area is $0.581 \mathrm{~cm}^{2}$; the moment of inertia is $0.0269 \mathrm{~cm}^{4}$, and the blade pitch at this position is $4.08 \mathrm{~cm}$. The shroud is set at the blade tip; the cross-sectional area is $0.945 \mathrm{~cm}^{2}$; the moment of inertia is $0.00709 \mathrm{~cm}^{4}$ for the tangential vibration and $0.781 \mathrm{~cm}^{4}$ for the axial vibration, and the blade pitch is $5.05 \mathrm{~cm}$.

\subsection{Test results}

3.2. 1 The resonant amplitude at the tangential natural frequency The calculated and measured results of the tangential amplitude at the tangential natural frequency are shown in Fig. 3. The measured result of the natural frequency was $124.4 \mathrm{~Hz}$ and the calculated result was $118.3 \mathrm{~Hz}$. The resonant amplitude was measured by excitation at an angle $50^{\circ}$ from the tangential direction and $6.6 \mathrm{~cm}$ from the blade tip of blade No. 3. The data needed to determine the exciting force is as follows: $e_{m}=0.19 \mathrm{~V}, f_{1}=62.2 \mathrm{~Hz}$, $S_{m}=S=8.4 \mathrm{~cm}^{2}, n=10$. Substituting these values in Eq. ( 8 ) and Eq. (10), the exciting force becomes $F_{1}=$ $0.56 \mathrm{~N}$.

To calculate the resonant amplitude, the logarithmic decrement was measured and the result was 0.0366 . When the resonant amplitude was calculated from the relationship in Eq. (4), the calculated values were compared with the measured ones, as shown in Fig. 3. The calculated results of the resonant amplitude agree well with the measured ones. At some measuring positions, the calculated values and the measured ones differed to some extent; in the worst case, the measured values were about twice the values of the calculated ones.

With regard to the natural frequency of the grouped blades, the calculated values mostly agreed with the measured ones, while the calculated and measured results of the resonant amplitude did not agree as well as the natural frequencies.

3.2. 2 The resonant amplitude at the axial natural frequency The calculated and measured results of the axial amplitude at the axial natural frequency are shown in Fig. 4. The resonant amplitude was measured during excitation in the axial direction $6.6 \mathrm{~cm}$ from the blade tip of blade No. 1. The measured result of the natural frequency was $193.6 \mathrm{~Hz}$, and the calculated frequency was $191.2 \mathrm{~Hz}$. The exciting force was $0.261 \mathrm{~N}$ and the logarithmic decrement was 0.029 .

The calculated results of the resonant amplitude agree well with the measured ones, At some measur. ing positions, a difference was observed between the calculated values and the measured ones; in the worst case, the measured values were about twice the value of the calculated ones. Thus, the results showed the same trends as in the case of the tangential natural frequency.

The test results with regard to one grouped blade are also reported. The calculated and measured results agreed to the same extent as similar test results with other grouped blades of different sizes.

With regard to the turbine blade, the principal axis direction of the cross section varies between the blade root and the blade tip. Therefore, the vibrating direction at the natural frequency differed according to the position along the blade. The direction of vibration at the axial natural frequency was calculated and measured. The results are shown in Fig. 5. The direction perpendicular to the direction of the minimum deflection is the vibrating direction. From the figure, it can be seen that the calculated values of the vibrating direction agree well with the measured ones.

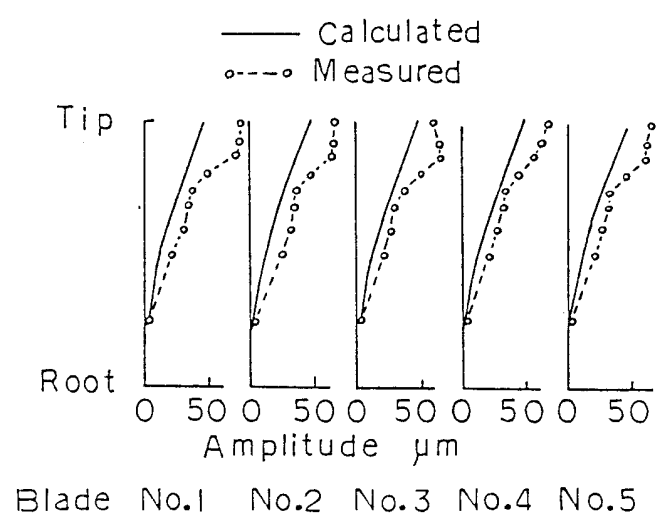

Fig. 3 Tangential amplitude at the tangential natural frequency

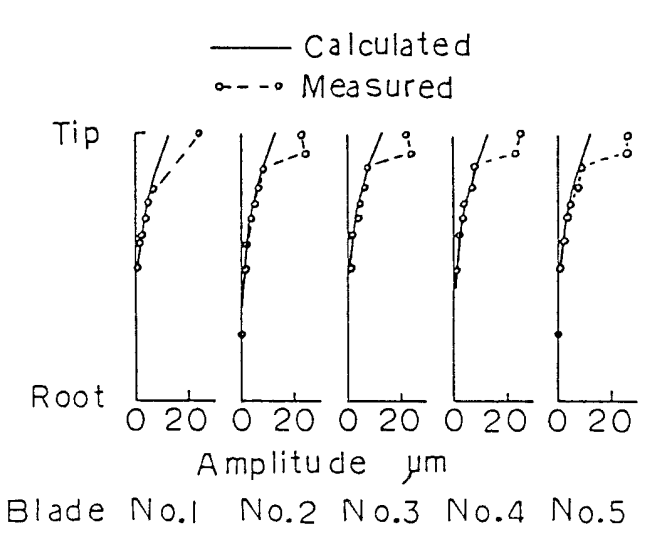

Fig. 4 Axial amplitude at the axial natural frequency 


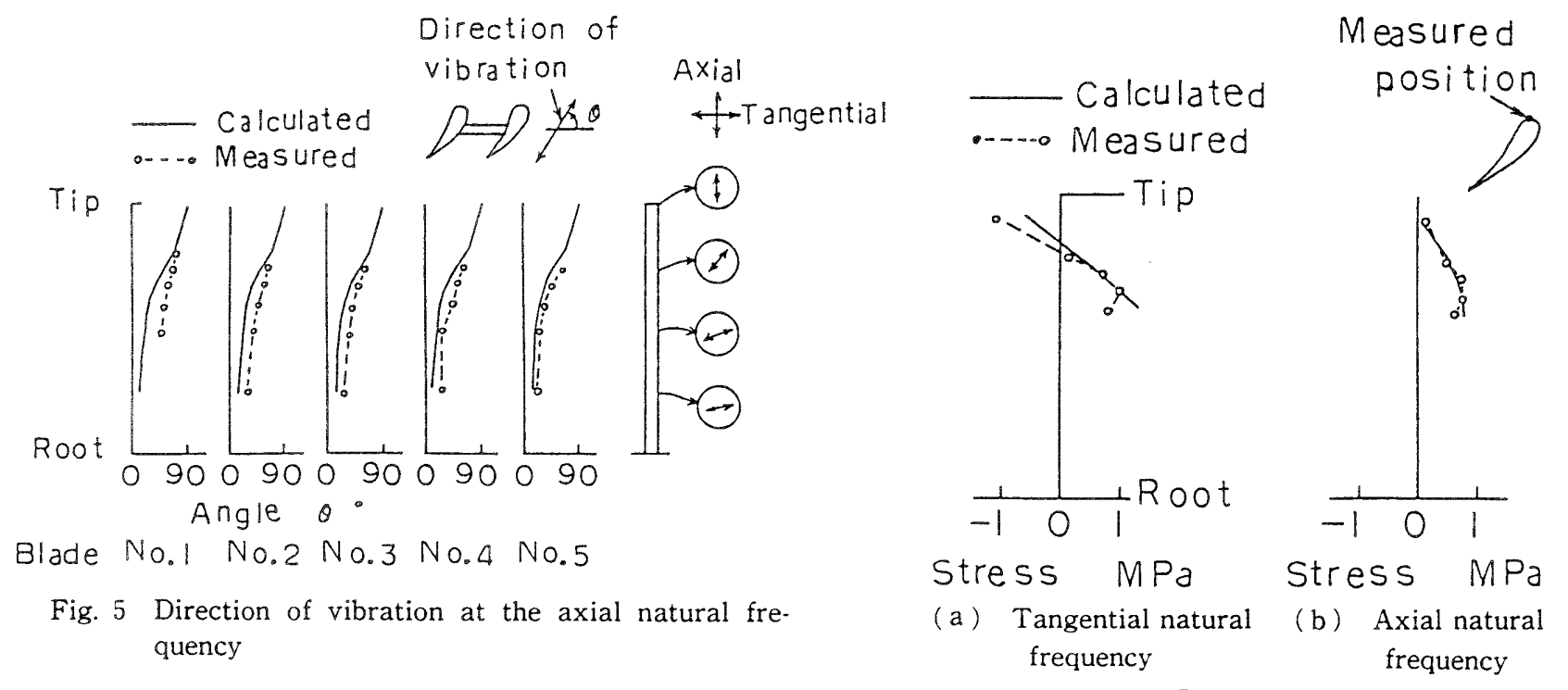

As shown in Fig. 5, the blade vibrates in the axial direction at the blade tip, and the vibrating direction changes towards the tangential direction closer to the blade root.

3.2.3 The resonant stress The calculated and measured values of the resonant stress are shown in Fig. 6. When the resonant amplitude was measured at the tangential and axial natural frequencies, the stress of the longitudinal direction of the No. 1 blade was measured. The results were compared with the calculated values. The calculated values agreed very well with the measured values.

\section{Conclusions}

In search of discrepancies between calculated and measured results of the resonant amplitude of turbine grouped blades, the resonant amplitude was calculated and measured with regard to five grouped blades. The calculated results of the resonant amplitude distribution at the natural frequency agreed well with the measured ones. At some measuring points, the calculated values and the measured ones differed, while in the worst case, the measured values were about twice the values of the calculated ones. With

regard to the direction of vibration and the resonant stress, the calculated values also agreed well with the measured ones.

Therefore, if the operational exciting force can be determined, the resonant vibration amplitude and stress of the turbine grouped blades can be predicted by calculation when designing the blades.

\section{References}

(1) Deak, A. L. and Baird, R. D., A Procedure for Calculating the Packet Frequencies of Steam Turbine Exhaust Blades, Trans. ASME, Ser. A, Vol. 85, No. 4 (1963), p. 324.

(2) See, for example, Michimura, S., Nagamatsu, A., Ikeuchi, T. and Shirai, M., Vibration Analysis on Bladed Disk Assemblies of Axial Flow Turbines, Trans. Jpn. Soc. Mech. Eng., (in Japanese), Vol. 47, No. 415, C (1981), p. 242.

(3) Prohl, M. A., A Method for Calculating Vibration Frequency and Stress of a Banded Group of Turbine Buckets, Trans. ASME, Vol. 80, No. 1, (1958), p. 169.

(4) Plunkett, R., Structural Damping, (1960), p. 117, Pergamon Press. 